\title{
Multigrid solution of 2D and 3D stress fields in contact mechanics of anisotropic inhomogeneous materials
}

\author{
Binbin Zhang ${ }^{\mathrm{a}, *}$, Hugo Boffy ${ }^{\mathrm{b}}$, Cornelis H. Venner ${ }^{\mathrm{a}}$ \\ ${ }^{\text {a }}$ Faculty of Engineering Technology, University of Twente, P.O. Box 217, 7500AE, Enschede, the Netherlands \\ ${ }^{\mathrm{b}}$ Engineering and Research Centre, SKF, Kelvinbaan 16, 3439 MT, Nieuwegein, the Netherlands
}

\section{A R T I C L E I N F O}

\section{Keywords:}

Multigrid method

Contact mechanics

Inhomogeneous material

Polycrystalline material

\begin{abstract}
A B S T R A C T
Increasing demands on performance of machines lead to severer operating conditions of rolling bearings, i.e. higher loads, less lubricant, thinner lubricant films. Under these conditions, the effects of inhomogeneity and anisotropy on the fatigue life become more important. Accurate prediction of such effects requires detailed surface pressure and subsurface stress calculations. For practically relevant 3D cases with realistic grain sizes, this can only be done with very efficient numerical solution methods. In this paper, multigrid techniques are demonstrated to yield the required performance. The influence of inclusions, crystal orientation and roughness on the Von Mises stress distribution is investigated. The algorithm is suited for subsurface material analysis and optimization as well as for computational diagnostics using image analysis.
\end{abstract}

\section{Introduction}

Increasingly critical design implies higher operational requirements for rolling bearings in many applications. As a result, further improvement of bearing life is a key target for rolling bearing analysis, design and development. Bearing damage can be caused by external working conditions (e.g. load, speed, temperature, lubrication condition, corrosion) and by intrinsic bearing properties (e.g. surface finish, coating, non-metallic inclusions, material anisotropy, residual stress). Even when a rolling bearing is properly installed and well lubricated, rolling contact fatigue (RCF) may occur initiated at the surface or in the subsurface after millions of load cycles.

Fatigue of rolling bearings can be classified into two types: subsurface-initiated fatigue and surface-initiated fatigue. For the subsurface-initiated fatigue, the Lundberg-Palmgren fatigue theory [1] presumes that the large orthogonal shear stress and a weak point in the material result in the initiation of fatigue crack. Later, Ioannides and Harris [2] extended the fatigue theory by introducing an additional material parameter. Recently, the surface-originated damage was explicitly formulated into the basic fatigue equations of rolling contact by Morales-Espejel et al. [3]. Performance factors were introduced into the new model, which makes it possible to target specific bearing features and operating conditions. Surface-initiated fatigue can be decreased by using better lubricants, surface heat treatment and better surface finish. Subsurface-initiated fatigue is much harder to control as it involves the local subsurface material composition and behavior. To model fatigue life well, analysis of the subsurface stress field in the bearing material induced by the contact pressure is needed. In the tribology literature, many publications have investigated the influence of surface defect/ roughness, coating, inclusions, and anisotropy on subsurface stresses in bearing material. Relatively recent examples are the Voronoi finite element model was used for damage mechanics by Warhadpande and Sadeghi [4] who observed that surface defects have a significant effect on RCF life in heavily loaded lubricated conditions. Considering surface coating, subsurface inclusions and surface roughness, Dong et al. [5] developed an approach for computing the stress field distribution. In their study, both inclusions and coating were homogenized using the Eshelby's equivalent inclusion method. Moghaddam et al. [6] studied the influence of non-metallic inclusions on butterfly wing initiation and crack formation. Their results showed that the stiffness and location of an inclusion have a significant effect on RCF. Slack et al. [7] investigated the influence of inclusions on an elastohydrodynamically lubricated (EHL) line contact. They found that the subsurface inclusions changed the EHL pressure and film thickness profiles. Morales-Espejel et al. [8] used a rapid micro-EHL methodology combined with a multigrid method for stress calculation and surface deformation [9] to study surface fatigue with heterogeneous material under rough lubricated rolling-sliding contact. The inhomogeneities near contact surface affect pressure and subsurface stress distribution. Minimizing the distribution of inhomogeneous material in bearing material can be

\footnotetext{
* Corresponding author.

E-mail address: mezbinbin@163.com (B. Zhang).
} 
achieved through advanced manufacturing processes. However, the anisotropy is an intrinsic property of the bearing material. It should be noted that the anisotropy of bearing material is scale dependent. On a macro scale, RCF is less sensitive to grain size and orientation. On a micro scale, its effect cannot be ignored. Noyel et al. [10] developed a granular cohesive model for RCF analysis. They found that RCF was influenced by anisotropic elasticity. Paulson and Sadeghi [11] developed a fully coupled polycrystalline anisotropic EHL model to investigate rolling contact fatigue. It was found that the anisotropy leads to stress components variation and then life scatter.

To model the stress concentration areas in anisotropic and inhomogeneous material under load in a theoretical/numerical study requires very detailed grids to be able to describe the small scale variations and obtain accurate results. To achieve this within acceptable computational times requires a highly efficient method. In Refs. $[12,13]$, Boffy et al. applied multigrid method to the 3D stress filed solution in heterogeneous and strong heterogeneous material with given contact pressure distribution. It is proved the developed multigrid algorithm possess high accuracy and efficiency. Later, it was extended to 3D contact mechanics with heterogeneous material [14]. In this paper, the multigrid method is demonstrated to be very well suited to solve the computationally even more demanding problem of contact mechanics and subsurface stress field for bearing material with heterogeneous properties on the very local scale of grains including anisotropy.

\section{Methods}

\subsection{Theoretical model}

The problem considered is the contact of a rigid indenter and an elastic body with anisotropic inhomogeneous material. A rectangular (2D) and cubic (3D) domains are considered.

\subsubsection{Interior equations}

The computational task can be described as the solution of the unknown displacements in a 2D or 3D domain from the stress equilibrium equations:

$$
\left\{\begin{array} { l } 
{ \frac { \partial \sigma _ { x x } } { \partial x } + \frac { \partial \sigma _ { x y } } { \partial y } = 0 } \\
{ \frac { \partial \sigma _ { x y } } { \partial x } + \frac { \partial \sigma _ { y y } } { \partial y } = 0 }
\end{array} \text { or } \left\{\begin{array}{l}
\frac{\partial \sigma_{x x}}{\partial x}+\frac{\partial \sigma_{x y}}{\partial y}+\frac{\partial \sigma_{x z}}{\partial z}=0 \\
\frac{\partial \sigma_{x y}}{\partial x}+\frac{\partial \sigma_{y y}}{\partial y}+\frac{\partial \sigma_{y z}}{\partial z}=0 \\
\frac{\partial \sigma_{x z}}{\partial x}+\frac{\partial \sigma_{y z}}{\partial y}+\frac{\partial \sigma_{z z}}{\partial z}=0
\end{array}\right.\right.
$$

where $\sigma_{i j}=C_{i j k l} \varepsilon_{k l}$. Details of the stress-strain tensor are given in appendix A. The stress-strain tensor in a local coordinate system (single grain) is still a relatively sparse matrix. However, in the global coordinate system needed to describe the complete problem and equations the tensor becomes full. This implies additional terms in the equations to be solved, the nature and spatial variation of which may adversely affect convergence behavior of an iterative numerical solution algorithm.

\subsubsection{Top boundary equations}

The unknown displacements and contact pressure of top boundary can be solved with Eqs. (2)-(4) [14].

For 2D:

$\sigma_{x y}=0$

$h(x)=h_{0}+g(x)-v(x, y=0)=0$

$\sigma_{y y}+p=0$

For 3D:

$\sigma_{x z}=0 \quad \sigma_{y z}=0$ $h(x, y)=h_{0}+g(x, y)-w(x, y, z=0)=0$

$$
\sigma_{z z}+p=0
$$

In Eq. (3), $g(x)$ and $g(x, y)$ represent the shape of the indenters. Eqs. (2)-(4) are used to solve the top boundary displacements and pressure distribution for the contact problem satisfying the Hertz-SignoriniMoreau condition, Eq. (5).

$p \geq 0 \quad h \geq 0 \quad p h=0$

This implies that for the indenter in contact with the elastic body $p>0$ and $h=0$. When the gap is open $h>0$, Eq. (3) cancels with $p=0$, and Eqs. (2) and (4) are used to calculate the top boundary displacements, i.e. stress free boundary condition.

Finally, the applied load $F$ should be balanced by the contact pressure:

$\int p(x)=F$ or $\quad \iint p(x, y)=F$

This equation determines the value of $h_{0}$.

\subsubsection{Vertical and bottom boundary equations}

The bottom boundary is specified as zero displacement (Dirichlet) and the vertical boundaries are assumed to be stress free (Neumann). Special attention should be paid to the intersections. Here, the problem is detailed for the 3D case, see Fig. 1. Normal and shear stresses are used to determine the displacements $u, v, w$ of the boundaries. At the intersection of the boundary planes (e.g. green line in Fig. 1), the conditions of the both planes should be satisfied. At the corner points (e.g. red point in Fig. 1), the sum of the stresses is imposed to be zero [15].

\subsection{Numerical technique}

The equations are discretized on a uniform grid using a finite difference approach, see Appendix A. The discrete equations are solved using multigrid techniques $[9,14]$. An iterative process is designed using a Gauss-Seidel (consecutive) relaxation scheme for the displacement equations with special treatment for the boundary equations to ensure a good error smoothing which is essential for a multigrid solver. For the top boundary, the complementarity equation for the contact pressure is solved simultaneously with the prescribed (zero) shear stress conditions in a pointwise collective boundary relaxation.

The key idea of the multigrid method is to overcome the slow grid dependent convergence of the relaxation process by using a coarser grid to solve smooth error components. Applied recursively using multiple coarser grids so that each error component is solved on a grid at which this can be done most efficiently, a high grid independent convergence rate is achieved, allowing the solution of problems with dense grids very efficiently. Multigrid techniques enable solving much larger problems with the available computational power. Further details are given in Refs. [9,14].

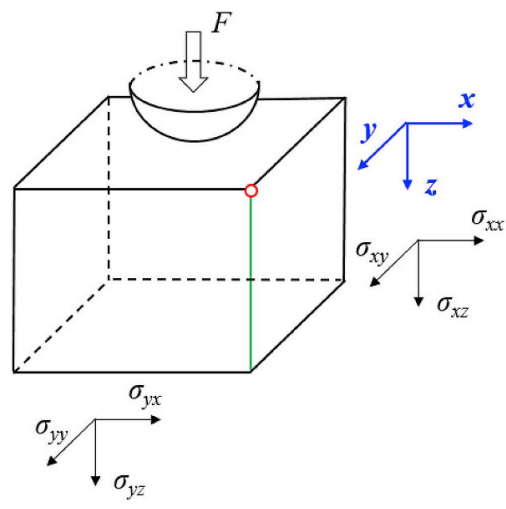

Fig. 1. Schematic graph of 3D elastic contact. 
Table 1

Stiffness matrix components of Ferrite.

\begin{tabular}{ll}
\hline Elastic constant & Stress (GPa) \\
\hline$c_{11}$ & 237 \\
$c_{12}$ & 141 \\
$c_{44}$ & 116 \\
\hline
\end{tabular}

Table 2

Material and contact parameters.

\begin{tabular}{ll}
\hline Parameter & Value \\
\hline$E$ & $206 \mathrm{GPa}$ \\
$\nu$ & 0.3 \\
$R$ & $0.01 \mathrm{~m}$ \\
$p_{\mathrm{Hz}}$ & $1 \mathrm{Gpa}$ \\
$b(2 \mathrm{D})$ & $69.4 \mu \mathrm{m}$ \\
$b$ (3D) & $88.7 \mu \mathrm{m}$ \\
\hline
\end{tabular}

\subsection{Solver performance}

The computational performance for the $3 \mathrm{D}$ problem is shown in this section. In the first example, homogeneous anisotropic ferrite is considered. The elastic constants are given in Table 1. The anisotropy ratio A (see definition in Appendix A) for ferrite is 2.4. The elastic stiffness matrix of the single grain is rotated based on Eq. (7) [16].

$C_{\text {global }}=R(\alpha) R(\beta) R(\gamma) C_{\text {local }}(R(\alpha) R(\beta) R(\gamma))^{T}$

where $\alpha, \beta, \gamma$ are the three Euler angles. A grid with $513^{3}$ points is used, and 5 grid levels in the multigrid algorithm. The material and contact parameters are given in Table 2. The problem is solved using a computational domain [-3a:3a, -3a:3a, 0:6a], with a the Hertzian dry contact radius for uniform isotropic material.

The residual for each of the discretized equations is defined by:

res $=\sum_{i, j, k}\left|\frac{f(i, j, k)-L u(i, j, k)}{N_{x} N_{y} N_{z}}\right|$

The convergence of the solver is monitored by the reduction of the L1 norm of the residual as a function of the number of multigrid $\mathrm{W}$ cycles, see Fig. 2. The figure shows that the developed multigrid algorithm exhibits uniform fast convergence for each of the three displacements equations and the contact condition.

This excellent convergence behavior is essentially maintained for the anisotropic problem. This is illustrated in Fig. 3 for the case of ferrite with some 4500 grains having randomly varying Euler angles $\alpha$, $\beta, \gamma$, ranging from 0 to $\pi / 2$. In terms of real dimensions for the given conditions, the average grain diameter is about $25 \mu \mathrm{m}$ [10]. In Fig. 3, the black, red and blue lines represent the residuals for the displacement variables $u, v$ and $w$ respectively. The pink line represents the residual of the force balance condition. Note that each equation

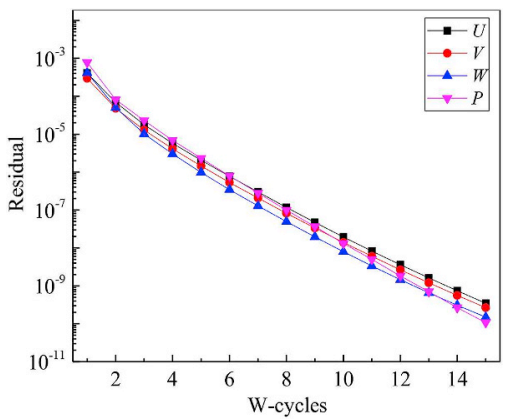

Fig. 2. Residual as a function of cycles (homogeneous ferrite), $513^{3}$ grid points, 5 multigrid levels.

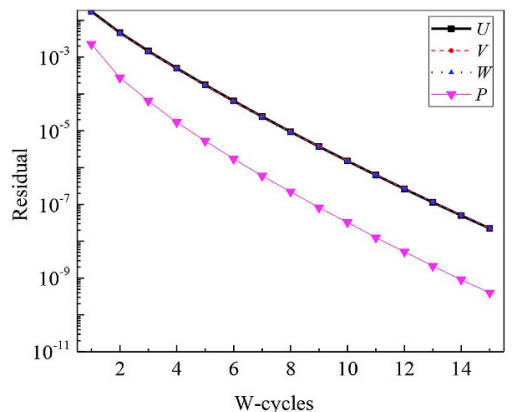

Fig. 3. Function residual as a function of cycles (about 4500 grains).

converges at the same speed with an order of magnitude error reduction per two W-cycles which in general is sufficient to achieve an error below the discretization error that is made anyway. The computations were done on a computer with an Intel X5650 CPU at $2.66 \mathrm{GHz}$, using a single core. The wall clock time for two cycles is about $89 \mathrm{~min}$ and for 10 cycles about $7 \mathrm{~h}$. In general, only one or two W-cycles are needed to solve the problem with an error below discretization error.

The results shown in Fig. 3 demonstrate that the developed solver is well suited for realistic material topologies. Detailed results for some 2D and 3D cases in terms of computed surface pressure and subsurface stresses will be shown in the next section.

\section{Results}

In this section, the influence of inclusions (heterogeneous material), anisotropy and surface roughness on the Von Mises stress distribution is illustrated. The average size of the Voronoi cells is $25 \mu \mathrm{m}$. First, as a reference a case of an isotropic material case in $2 \mathrm{D}$ is studied with some of the cells having smaller or larger elastic modulus. Material properties and contact pressure are given in Table 2.

The results are presented in dimensionless form taking the Hertz contact parameters as a reference (see nomenclature), i.e. the coordinates $x, y, z$, displacements $u, v, w$ and gap distance $h$ are dimensionalized by Hertzian half contact width $b$. The pressure, elastic stiffness matrix and stress are scaled by maximum Hertzian contact pressure. The dimensionless computational domain used is $[-3: 3,0: 6]$ for $2 \mathrm{D}$ and $[-3: 3,-3: 3,0: 6]$ for $3 \mathrm{D}$.

Fig. 4(a) shows a 2D Voronoi tessellation in which the black and green regions represent randomly selected inclusions. Fig. 4 (b) $\sim$ (d) show the top pressure distribution, gap distance and subsurface Von Mises stress respectively. Fig. 4(b) and (c) give results when the black and green regions in Fig. 4(a) represent inclusions four times softer or harder than the bulk material. Fig. 4(d) shows the result when the black regions are treated as hard inclusions and the green regions as soft. The Von Mises stress shows that both soft and hard inclusions act as local stress raisers. Hard inclusions result in larger local stress concentration, which increases $21 \%$ compared to the result of soft inclusions. When inclusions are sufficiently close to the surface, they affect the contact pressure distribution [17]. The local stress concentration is detrimental for the fatigue life of bearing material. Compared to homogeneous material, cracks are likely to initiated at/near the inclusions [6].

Fig. 5 shows the influence of the load (relative to the homogeneous case) on the maximum Von Mises stress value and its location for the case studied in Fig. 4 (d). For low loads (below 1.0), the maximum Von Mises stress increases with load and its location remains at one of the near surface inclusions. For higher loads (larger than 1.0), the location of the maximum Von Mises stress changes to deeper inclusions. With the increase of the load, the variation of maximum Von Mises stress becomes steeper. In the end, the value of maximum Von Mises stress is around eight times that of the lowest load case. Thus the potential possibility of crack formation is much higher with a heavy load in the 


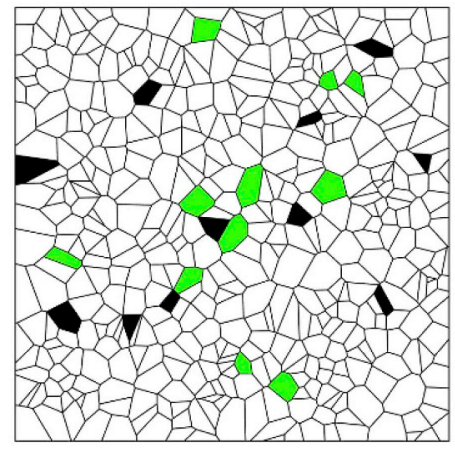

(a) 2D Voronoi tessellation

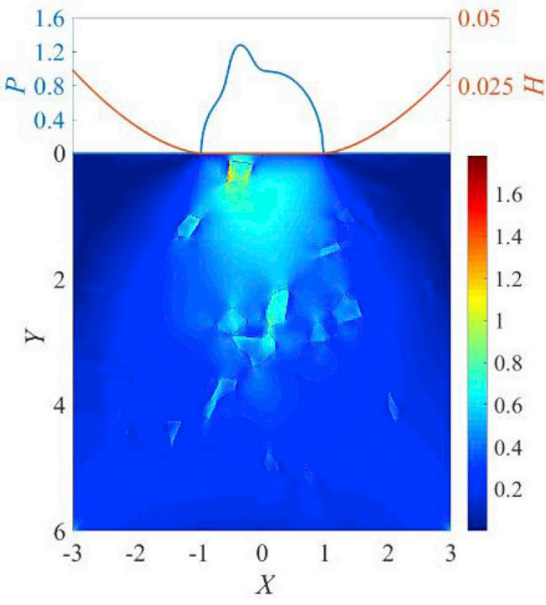

(c) $E_{\mathrm{i}}=4 E$

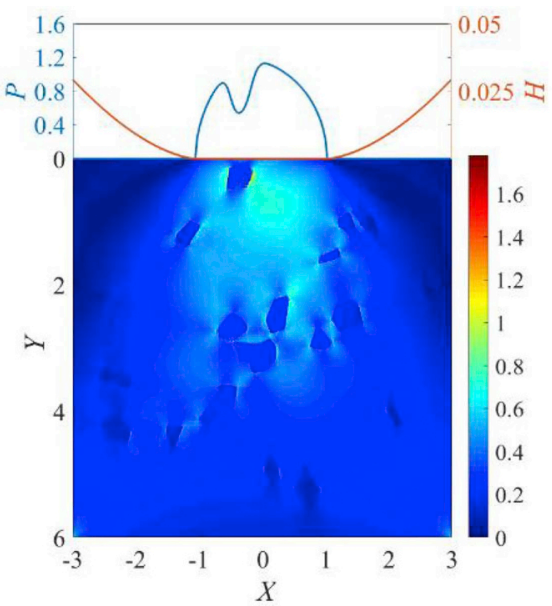

(b) $E_{\mathrm{i}}=0.25 E$

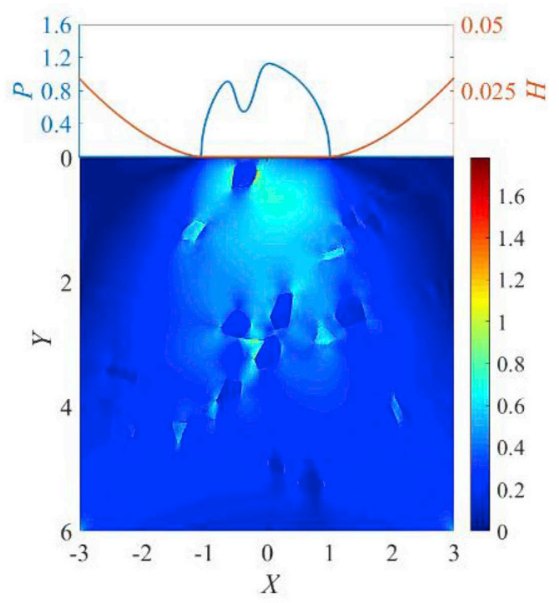

(d) $\left(E_{\mathrm{i}}\right)_{\text {green }}=0.25 E$ and $\left(E_{\mathrm{i}}\right)_{\text {black }}=4 E$

Fig. 4. Voronoi tessellation and top boundary pressure, gap distance and Von Mises stress distribution.

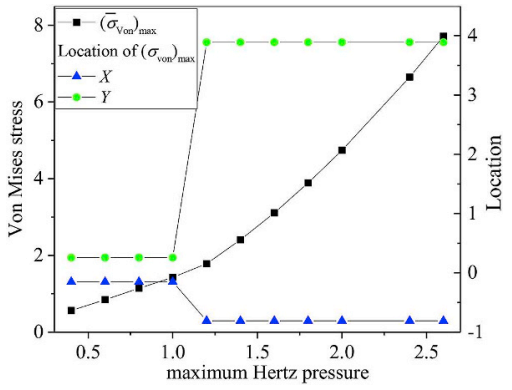

Fig. 5. The influence of load on the maximum Von Mises stress.

presence of inclusions.

Next, a more challenging 3D problem is considered for which the solver was developed, i.e. anisotropic material and where the efficiency of multigrid method is really needed. Fig. 6 (a) shows the 3D Voronoi tessellation [18]. The parameters of grain elastic stiffness matrix in local coordinate system are given in Table 1. Material and contact parameters are as given in Table 2. Each grain is assigned three random Euler angles $\alpha, \beta, \gamma$, ranging from 0 to $\pi / 2$. From local coordinate system to global coordinate system, the rotation of the elastic stiffness matrix is based on Eq. (7).

The Von Mises stress in the central $X Z$ and $Y Z$ planes is shown in
Fig. 6 (b) and (c) respectively. Note that a difference of rotation angles between grains in the anisotropic material represents different stiffness properties in the global coordinate system. As a result, some grains behave as hard inclusions whereas others behave as soft inclusions. Stress concentrations appear around grain boundaries with large rotation angle variation.

Fig. 7 shows the top surface pressure distribution. The change of local grain orientation induces significant variation of the pressure relative to the homogeneous anisotropic case. Pressure fluctuations appear near the grain boundaries. In order to achieve a smooth transition between grain boundaries for stress field and extend the fatigue life of rolling bearings, the variation of local grain orientation should be reduced.

Finally, it is demonstrated that the developed algorithm can efficiently deal with the combined case of a complex contact problem with local separation due to roughness and material anisotropy.

Fig. 8 shows the influence of an isotropic harmonic surface waviness (with wavelength $45.68 \mu \mathrm{m}$ and amplitude $0.069 \mu \mathrm{m}$ ) on the contact pressure and the Von Mises stress distribution. It can be seen from the contact pressure (Fig. 8(a)) that the relatively large amplitude of the waviness leads to multiple isolated contact islands at the peaks. Regarding the behavior of the Von-Mises stress, it leads to shallow concentrations, which are detrimental for the contact bodies. To avoid these, the rollers and raceway of rolling bearings should be manufactured with good surface finish. This result demonstrates that the 


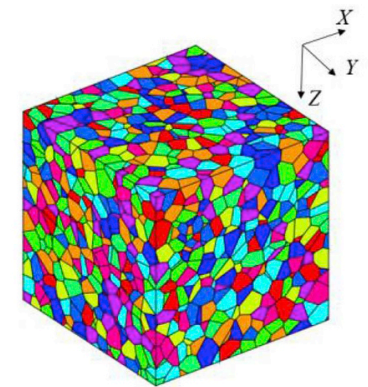

(a) 3D Voronoi tessellation

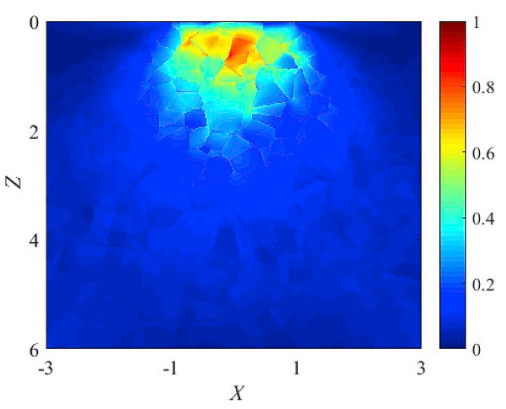

(b) $X Z$ plane $(Y=0)$

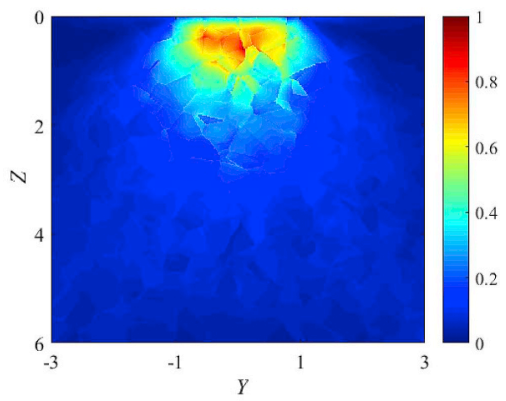

(c) $Y Z$ plane $(X=0)$

Fig. 6. 3D Voronoi tessellation and Von Mises stress distribution.

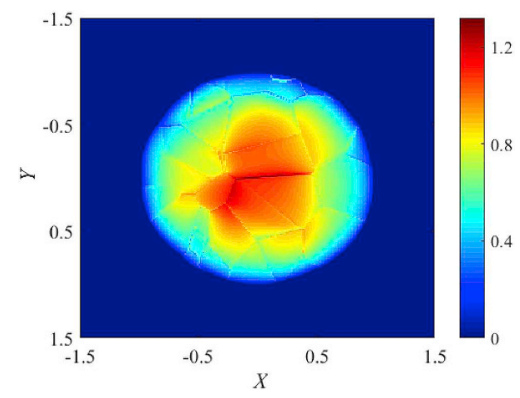

Fig. 7. Top surface pressure distribution.

developed algorithm is fully suited for extensive studies of anisotropic material behavior in contact applications.

\section{Discussion}

Both soft and hard inclusions affect the subsurface stress distribution (Fig. 4 (b)-(d)). The local stress variation is unbeneficial for fatigue life of rolling bearings. After millions of load cycles, cracks may be easier to occur around these inclusions. And the cracks will gradually grow in the horizontal and vertical directions and result in material spalling eventually. The influence of anisotropy on the subsurface stress field is not obvious as that is shown in the inclusions case. With the

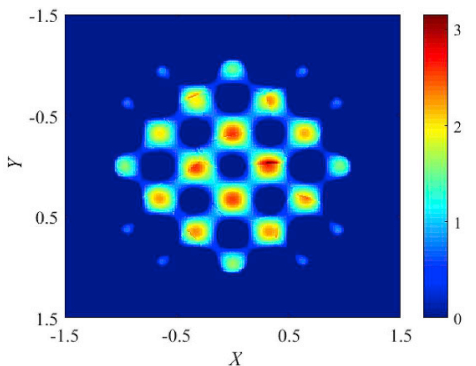

(a) Pressure distribution of rough contact

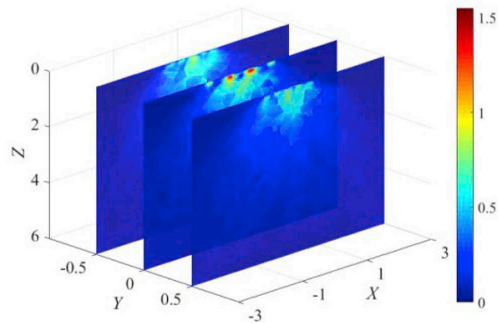

(b) Von Mises stress of $X Z$ planes

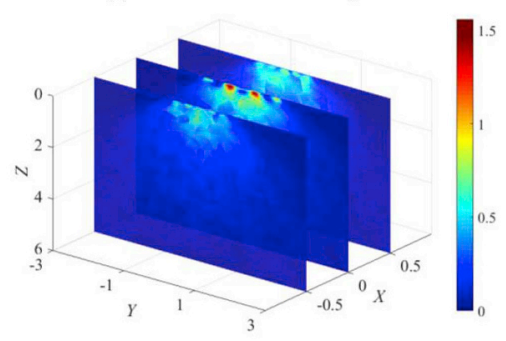

(c) Von Mises stress of $Y Z$ planes

Fig. 8. Effect of roughness on pressure and Von Mises stress distribution.

decrease of grain size and local anisotropy variation, its effect will become tinier.

Material inclusions and anisotropy also affect contact pressure distribution. When contact bodies are lubricated, the EHL pressure distribution can be affected by material inclusions and anisotropy as well $[7,11]$. Thus in order to extend the fatigue life of rolling bearings, the size and number of inclusions and local anisotropy variation in bearing material should be minimized as much as possible by modern manufacturing technology.

\section{Conclusions and future research}

A multigrid solver has been developed for the numerical solution of the contact problem and subsurface stress field calculation in inhomogeneous and anisotropic materials. Excellent performance has been demonstrated for a 2D problem with inclusions and a 3D problem with grain elasticity (anisotropy) and surface roughness. The developed method can solve problems with high spatial resolution allowing detailed study of local variations in the material. From the results presented, it is concluded that:

1. Material inclusions and anisotropy significantly affect subsurface stress and contact pressure distribution, where these effects significantly increase with increasing load.

2. Surface roughness brings stress concentration point near the contact surface, possibly augmenting material anisotropic effects and are therefore detrimental for rolling bearing fatigue life.

The high efficiency and accuracy of the developed algorithm allows detailed simulations even on relatively standard personal computers. 
The algorithm can be exploited for many other studies such as: material optimization on a local scale with the aim to extend the fatigue life of rolling bearings. The determination of criticality criteria for local material variations/inclusions, and computational diagnostics based on tomographic images. Finally, the developed method can relatively easily be coupled with EHL and study the mutual influence between anisotropic inhomogeneous material and EHL pressure [8,11].

\section{Acknowledgements}

The authors would like to thank Dr. Stefan Lammens, Director of SKF Research and Technology Development, for his kind permission to publish this article. This work is sponsored by SKF Research and Technology Development, the Netherlands. The authors would also like to thank Professor A.A. Lubrecht of INSA-Lyon, France, for the helpful discussions. Mr. Binbin Zhang would like to acknowledge the China Scholarship Council (CSC) for providing the PhD scholarship.

\section{Nomenclature}

A anisotropy material ratio, (-)

$b \quad$ half width of Hertzian contact (m)

$c_{11}, c_{12}, c_{44}$ elastic constants for cubic material, (Pa)

$C, C_{\text {global }}, C_{\text {local }}$ elastic stiffness matrix, $(\mathrm{Pa})$

E Young's modulus, (Pa)

$E_{\mathrm{i}} \quad$ Young's modulus of inlusion, $(\mathrm{Pa})$

$F \quad$ applied load, $(\mathrm{N})$ for 3D problem, $(\mathrm{N} / \mathrm{m})$ for $2 \mathrm{D}$ problem

$g(x), g(x, y)$ shape of cylinder and sphere, (m)

$h \quad$ gap between rigid indenter and top surface, (m)

$h_{x}, h_{y}, h_{z}$ mesh space in $x, y, z$ directions, (m)

$H \quad$ dimensionless gap height, $h / b$

$h_{0} \quad$ height of rigid indenter, $(\mathrm{m})$

$p \quad$ contact pressure, $(\mathrm{Pa})$

$P \quad$ dimensionless contact pressure, $p / p_{\mathrm{Hz}}$

$R \quad$ radius of rigid indenter, (m)

$p_{\mathrm{Hz}} \quad$ maximum Hertzian pressure for rigid indenter contacts with elastic body, (Pa)

$u, v, w \quad$ displacements in $x, y, z$ directions, (m)

$U, V, W$ dimensionless displacements, $u / b, v / b, w / b$

$x, y, z \quad$ coordinate system, (m)

$X, Y, Z \quad$ dimensionless coordinate system, $x / b, y / b, z / b$

$\sigma_{x x}, \sigma_{y y}, \sigma_{z z}$ normal stress, (Pa)

$\sigma_{x y}, \sigma_{x z}, \sigma_{y z}$ shear stress, $(\mathrm{Pa})$

$\bar{\sigma}_{\mathrm{von}} \quad$ dimensionless Von Mises stress, $\sigma_{\mathrm{von}} / p_{\mathrm{Hz}}$

$\alpha, \beta, \gamma \quad$ Euler rotation angle, (rad)

$\varepsilon \quad$ strain, $(-)$

$v \quad$ Poisson's ratio, $(-)$

$\lambda \quad$ Lame modulus, $(\mathrm{Pa})$

$\mu \quad$ Shear modulus, $(\mathrm{Pa})$

\section{Appendix A. Discrete equations}

The Voigt stress-strain relation is given in Eq. (9)

$$
\left[\begin{array}{c}
\sigma_{x x} \\
\sigma_{y y} \\
\sigma_{z z} \\
\sigma_{y z} \\
\sigma_{x z} \\
\sigma_{x y}
\end{array}\right]=C\left[\begin{array}{c}
\varepsilon_{x x} \\
\varepsilon_{y y} \\
\varepsilon_{z z} \\
2 \varepsilon_{y z} \\
2 \varepsilon_{x z} \\
2 \varepsilon_{x y}
\end{array}\right]
$$

where $C$ is the elastic stiffness matrix, for isotropic material, it has the form as Eq. (10)

$C=\left[\begin{array}{cccccc}\lambda+2 \mu & \lambda & \lambda & 0 & 0 & 0 \\ \lambda & \lambda+2 \mu & \lambda & 0 & 0 & 0 \\ \lambda & \lambda & \lambda+2 \mu & 0 & 0 & 0 \\ 0 & 0 & 0 & \mu & 0 & 0 \\ 0 & 0 & 0 & 0 & \mu & 0 \\ 0 & 0 & 0 & 0 & 0 & \mu\end{array}\right]$

where $\lambda=\frac{\nu E}{(1+\nu)(1-2 v)}, \mu=\frac{E}{2(1+\nu)}$. For anisotropic material (cubic), it is given in Eq. (11). 
$C=\left[\begin{array}{cccccc}c_{11} & c_{12} & c_{12} & 0 & 0 & 0 \\ c_{12} & c_{11} & c_{12} & 0 & 0 & 0 \\ c_{12} & c_{12} & c_{11} & 0 & 0 & 0 \\ 0 & 0 & 0 & c_{44} & 0 & 0 \\ 0 & 0 & 0 & 0 & c_{44} & 0 \\ 0 & 0 & 0 & 0 & 0 & c_{44}\end{array}\right]$

The difference of the two matrices is the value of $c_{44}$, which determines the anisotropy ratio $A=2 c_{44} /\left(c_{11}-c_{12}\right)$ to be different from 1 . Note that due to this difference, the matrix $C$ in global coordinates is a full matrix:

$C=\left[\begin{array}{llllll}c_{1111} & c_{1122} & c_{1133} & c_{1123} & c_{1113} & c_{1112} \\ c_{2211} & c_{2222} & c_{2233} & c_{2223} & c_{2213} & c_{2212} \\ c_{3311} & c_{3322} & c_{3333} & c_{3323} & c_{3313} & c_{3312} \\ c_{2311} & c_{2322} & c_{2333} & c_{2323} & c_{2313} & c_{2312} \\ c_{1311} & c_{1322} & c_{1333} & c_{1323} & c_{1313} & c_{1312} \\ c_{1211} & c_{1222} & c_{1233} & c_{1223} & c_{1213} & c_{1212}\end{array}\right]$

Below, the detailed equations and discretization are given for the 3D anisotropic case.

\section{A.1 Interior equations}

According to the above description, the detailed 3D stress equilibrium equation in $x$ direction can be written as:

$\frac{\partial}{\partial x}\left(c_{1111} \frac{\partial u}{\partial x}+c_{1122} \frac{\partial v}{\partial y}+c_{1133} \frac{\partial w}{\partial z}+c_{1123}\left(\frac{\partial v}{\partial z}+\frac{\partial w}{\partial y}\right)+c_{1113}\left(\frac{\partial u}{\partial z}+\frac{\partial w}{\partial x}\right)+c_{1112}\left(\frac{\partial u}{\partial y}+\frac{\partial v}{\partial x}\right)\right)+$

$\frac{\partial}{\partial y}\left(c_{1211} \frac{\partial u}{\partial x}+c_{1222} \frac{\partial v}{\partial y}+c_{1233} \frac{\partial w}{\partial z}+c_{1223}\left(\frac{\partial v}{\partial z}+\frac{\partial w}{\partial y}\right)+c_{1213}\left(\frac{\partial u}{\partial z}+\frac{\partial w}{\partial x}\right)+c_{1212}\left(\frac{\partial u}{\partial y}+\frac{\partial v}{\partial x}\right)\right)+$

$\frac{\partial}{\partial z}\left(c_{1311} \frac{\partial u}{\partial x}+c_{1322} \frac{\partial v}{\partial y}+c_{1333} \frac{\partial w}{\partial z}+c_{1323}\left(\frac{\partial v}{\partial z}+\frac{\partial w}{\partial y}\right)+c_{1313}\left(\frac{\partial u}{\partial z}+\frac{\partial w}{\partial x}\right)+c_{1312}\left(\frac{\partial u}{\partial y}+\frac{\partial v}{\partial x}\right)\right)=0$

The underlined terms in Eq. (13) directly follow from Eqs. (10) and (11). The other terms result from the matrix rotation from the local coordinate system to the global coordinate system. Obviously when the material is homogeneous anisotropic and the global coordinate system aligns with the direction of anisotropy, these terms will disappeared.

The equations are discretized using finite differences. The indices $i, j, k$ are used for $x, y, z$ direction respectively. The mesh size for each dimension is $h_{x}, h_{y}, h_{z}$. For the results shown above, a uniform mesh space is used. The discretization of the first term $\frac{\partial}{\partial x}()$ in Eq. (13) at grid point ( $i$, $j, k$ ) is given in Eq. (14).

$\frac{\left(c_{1111}\right)_{i+\frac{1}{2}, j, k} u_{i+1, j, k}-\left(\left(c_{1111}\right)_{i+\frac{1}{2}, j, k}+\left(c_{1111}\right)_{i-\frac{1}{2}, j, k}\right) u_{i, j, k}+\left(c_{1111}\right)_{i-\frac{1}{2}, j, k} u_{i-1, j, k}}{h_{x}^{2}}+$

$\frac{\left(c_{1122}\right)_{i+1, j, k}\left(v_{i+1, j+1, k}-v_{i+1, j-1, k}\right)-\left(c_{1122}\right)_{i-1, j, k}\left(v_{i-1, j+1, k}-v_{i-1, j-1, k}\right)}{4 h_{x} h_{y}}+$

$\frac{\left(c_{1133}\right)_{i+1, j, k}\left(w_{i+1, j, k+1}-w_{i+1, j, k-1}\right)-\left(c_{1133}\right)_{i-1, j, k}\left(w_{i-1, j, k+1}-w_{i-1, j, k-1}\right)}{4 h_{x} h_{z}}+$

$\frac{\left(c_{1123}\right)_{i+1, j, k}\left(v_{i+1, j, k+1}-v_{i+1, j, k-1}\right)-\left(c_{1123}\right)_{i-1, j, k}\left(v_{i-1, j, k+1}-v_{i-1, j, k-1}\right)}{4 h_{x} h_{z}}+$

$\frac{\left(c_{1123}\right)_{i+1, j, k}\left(w_{i+1, j+1, k}-w_{i+1, j-1, k}\right)-\left(c_{1123}\right)_{i-1, j, k}\left(w_{i-1, j+1, k}-w_{i-1, j-1, k}\right)}{4 h_{x} h_{y}}+$

$\frac{\left(c_{1113}\right)_{i+1, j, k}\left(u_{i+1, j, k+1}-u_{i+1, j, k-1}\right)-\left(c_{1113}\right)_{i-1, j, k}\left(u_{i-1, j, k+1}-u_{i-1, j, k-1}\right)}{4 h_{x} h_{z}}+$

$\frac{\left(c_{1113}\right)_{i+\frac{1}{2}, j, k} w_{i+1, j, k}-\left(\left(c_{1113}\right)_{i+\frac{1}{2}, j, k}+\left(c_{1113}\right)_{i-\frac{1}{2}, j, k}\right) w_{i, j, k}+\left(c_{1113}\right)_{i-\frac{1}{2}, j, k} w_{i-1, j, k}}{h_{x}^{2}}+$

$\frac{\left(c_{1112}\right)_{i+1, j, k}\left(u_{i+1, j+1, k}-u_{i+1, j-1, k}\right)-\left(c_{1112}\right)_{i-1, j, k}\left(u_{i-1, j+1, k}-u_{i-1, j-1, k}\right)}{4 h_{x} h_{y}}+$

$\frac{\left(c_{1112}\right)_{i+\frac{1}{2}, j, k} v_{i+1, j, k}-\left(\left(c_{1112}\right)_{i+\frac{1}{2}, j, k}+\left(c_{1112}\right)_{i-\frac{1}{2}, j, k}\right) v_{i, j, k}+\left(c_{1112}\right)_{i-\frac{1}{2}, j, k} v_{i-1, j, k}}{h_{x}^{2}}$

The discretization of the $\frac{\partial}{\partial y}()$ and the $\frac{\partial}{\partial z}()$ in Eq. (13) as well as the discretization of the equations for $y$ and $z$ directions in Eq. (1) can be done in the same way. 


\section{A.2 Boundary equations}

For the top boundary, without considering friction in $x$ and $y$ directions, when the indenter contacts the elastic body, the following equations are used:

$c_{1311} \frac{\partial u}{\partial x}+c_{1322} \frac{\partial v}{\partial y}+c_{1333} \frac{\partial w}{\partial z}+c_{1323}\left(\frac{\partial v}{\partial z}+\frac{\partial w}{\partial y}\right)+c_{1313}\left(\frac{\partial u}{\partial z}+\frac{\partial w}{\partial x}\right)+c_{1312}\left(\frac{\partial u}{\partial y}+\frac{\partial v}{\partial x}\right)=0$

$c_{2311} \frac{\partial u}{\partial x}+c_{2322} \frac{\partial v}{\partial y}+c_{2333} \frac{\partial w}{\partial z}+c_{2323}\left(\frac{\partial v}{\partial z}+\frac{\partial w}{\partial y}\right)+c_{2313}\left(\frac{\partial u}{\partial z}+\frac{\partial w}{\partial x}\right)+c_{2312}\left(\frac{\partial u}{\partial y}+\frac{\partial v}{\partial x}\right)=0$

$h_{0}+g(x, y)-w(x, y, z=0)=0$

$c_{3311} \frac{\partial u}{\partial x}+c_{3322} \frac{\partial v}{\partial y}+c_{3333} \frac{\partial w}{\partial z}+c_{3323}\left(\frac{\partial v}{\partial z}+\frac{\partial w}{\partial y}\right)+c_{3313}\left(\frac{\partial u}{\partial z}+\frac{\partial w}{\partial x}\right)+c_{3312}\left(\frac{\partial u}{\partial y}+\frac{\partial v}{\partial x}\right)+p=0$

The above four equations are solved to determine the displacements $u, v, w$ and the pressure $p$. When the indenter does not contact with the elastic body, i.e. the value of the third equation is larger than zero, the displacement $w$ is solved by the fourth equation with $p=0$. The discretization form for $\sigma_{x z}$ at grid point $(i, j, 0)$ is given in Eq. (19). The rules for this discretization are as follow: 1. the item with $u$ that exists before rotation uses second order forward discretization; 2. the items with $u$ that are introduced after rotation use second order central discretization; 3 . the items with $v$ and $w$ use second order central discretization in $x$ and $y$ directions and use first order discretization in $z$ direction. Eqs. (15), (16) and (18) can be discretized according to this rule. Note that for Eq. (16), $u$ in rule 1 and 2 is replace by $v$, and $v, w$ in rule 3 are replace by $u, w$. For Eq. (18), $u$ in rule 1 and 2 is replace by $w$, and $v, w$ in rule 3 are replace by $u, v$. The vertical boundaries are similar to the top boundary when the indenter does not contact the elastic body. And its discretization can be finished according to the top boundary.

$c_{1311} \frac{u_{i+1, j, 0}-u_{i-1, j, 0}}{2 h_{x}}+c_{1322} \frac{v_{i, j+1,0}-v_{i, j-1,0}}{2 h_{y}}+$

$c_{1333} \frac{w_{i, j, 0}-w_{i, j, 1}}{h_{z}}+$

$c_{1323}\left(\frac{v_{i, j, 0}-v_{i, j, 1}}{h_{z}}+\frac{w_{i, j+1,0}-w_{i, j-1,0}}{2 h_{y}}\right)+$

$c_{1313}\left(\frac{1.5 u_{i, j, 0}-2.0 u_{i, j, 1}+0.5 u_{i, j, 2}}{h_{z}}+\frac{w_{i+1, j, 0}-w_{i-1, j, 0}}{2 h_{x}}\right)+$

$c_{1312}\left(\frac{u_{i, j+1,0}-u_{i, j-1,0}}{2 h_{y}}+\frac{v_{i+1, j, 0}-v_{i-1, j, 0}}{2 h_{x}}\right)=0$

The discrete force balance equation reads:

$h_{x} h_{y} \sum_{i} \sum_{j} p_{i, j}=F$

\section{References}

[1] Lundberg G, Arvid P. Dynamic capacity of rolling bearings. Stockholm: Generalstabens litografiska anstalts förlag; 1947. p. 1-52.

[2] Ioannides E, Harris TA. A new fatigue life model for rolling bearings. J Tribol 1985;107(3):367-77https://doi.org/10.1115/1.3261081.

[3] Morales-Espejel GE, Gabelli A, De Vries AJ. A model for rolling bearing life with surface and subsurface survival-tribological effects. Tribol Trans 2015;58(5):894-906https://doi.org/10.1080/10402004.2015.1025932.

[4] Warhadpande A, Sadeghi F. Effects of surface defects on rolling contact fatigue of heavily loaded lubricated contacts. Proc IME J J Eng Tribol 2010;224(10):1061-77https://doi.org/10.1243/13506501JET785.

[5] Dong Q, Yang J, Wang X, Keer M, Zhou K. Heterogeneous structures with inhomogeneous inclusions under elastohydrodynamic lubrication contact with consideration of surface roughness. Proc IME J J Eng Tribol 2016;230(5):571-82https://doi.org/10.1177/1350650115606944.

[6] Moghaddam SM, Sadeghi F, Paulson K, Weinzapfel N, Correns M, Bakolas V, Dinkel M. Effect of non-metallic inclusions on butterfly wing initiation, crack formation, and spall geometry in bearing steels. Int J Fatigue 2015;80:203-15https://doi.org/ 10.1016/j.ijfatigue.2015.05.010.

[7] Slack TS, Raje N, Sadeghi F, Doll G, Hoeprich MR. EHL modeling for nonhomogeneous materials: the effect of material inclusions. J Tribol 2007;129(2):256-73https://doi.org/10.1115/1.2540234.

[8] Morales-Espejel GE, Boffy H, Venner CH. Effects of material heterogeneity on surface fatigue for rough lubricated rolling-sliding contacts. Proc IME J J Eng Tribol 2017;231(2):274-90https://doi.org/10.1177/1350650116650126.

[9] Venner CH, Lubrecht AA. Multi-level methods in lubrication. Amsterdam: Elsevier;
2000.

[10] Noyel JP, Ville F, Jacquet P, Gravouil A, Changenet C. Development of a granular cohesive model for rolling contact fatigue analysis: crystal anisotropy modelling. Tribol Trans 2016;59(3):469-79https://doi.org/10.1080/10402004.2015. 1087076.

[11] Paulson NR, Sadeghi F. EHL modeling of nonhomogeneous materials: the effects of polycrystalline anisotropy on RCF. Tribol Int 2017;112:137-46https://doi.org/10. 1016/j.triboint.2017.04.007.

[12] Boffy H, Baietto MC, Sainsot P, Lubrecht AA. An efficient 3D model of heterogeneous materials for elastic contact applications using multigrid methods. J Tribol 2012;134(2):021401https://doi.org/10.1115/1.4006296.

[13] Boffy H, Venner CH. Multigrid solution of the 3D stress field in strongly heterogeneous materials. Tribol Int 2014;74:121-9https://doi.org/10.1016/j.triboint. 2014.02.019.

[14] Boffy H, Venner CH. Multigrid numerical simulation of contact mechanics of elastic materials with 3D heterogeneous subsurface topology. Tribol Int 2015;92:233-45https://doi.org/10.1016/j.triboint.2015.06.015.

[15] Pérez-Ruiz JA, Luzón F, García-Jerez A. Simulation of an irregular free surface with a displacement finite-difference scheme. Bull Seismol Soc Am 2005;95(6):2216-31https://doi.org/10.1785/0120050014.

[16] Bower AF. Applied mechanics of solids. United States: CRC press; 2009.

[17] Zhang M, Zhao N, Wang Z, Wang Q. Efficient numerical method with a dual-grid scheme for contact of inhomogeneous materials and its applications. Comput Mech 2018:1-17https://doi.org/10.1007/s00466-018-1543-3.

[18] Herceg M, Kvasnica M, Jones CN, Morari M. Multi-parametric toolbox 3.0. European control conference. ECC; 2013. p. 502-10https://doi.org/10.23919/ECC 2013.6669862. 